

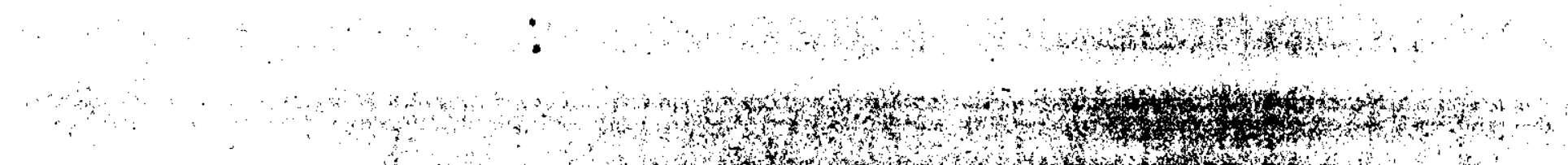

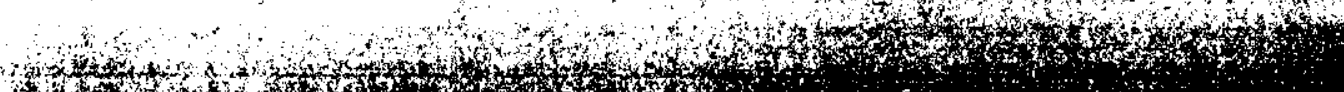

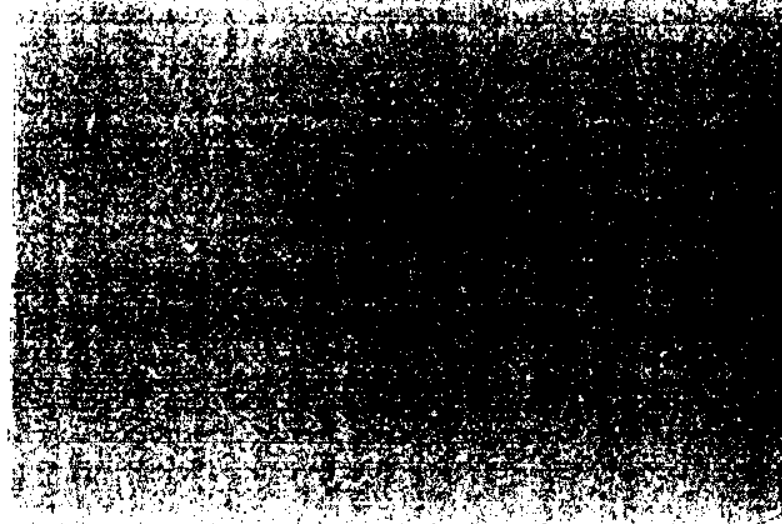

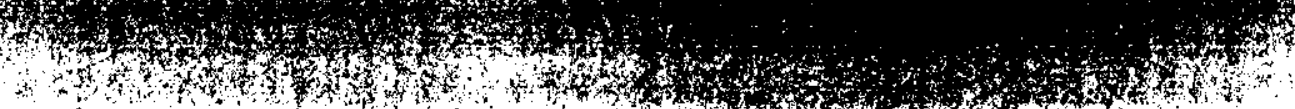

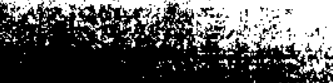

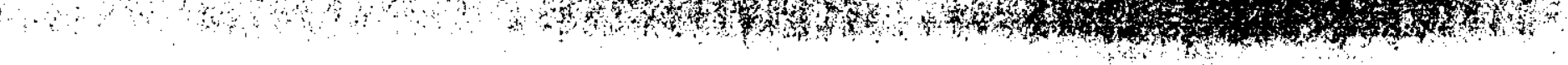

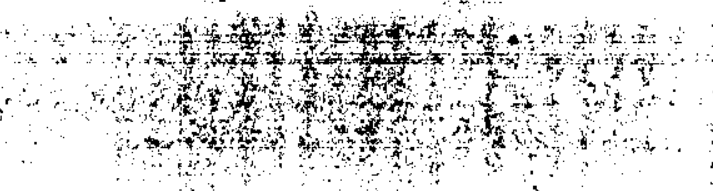

To be sure that your preprints are promptly included in the HIGH ENERGY PHYSICS INDEX send them to the following address (if possible by air mail) :

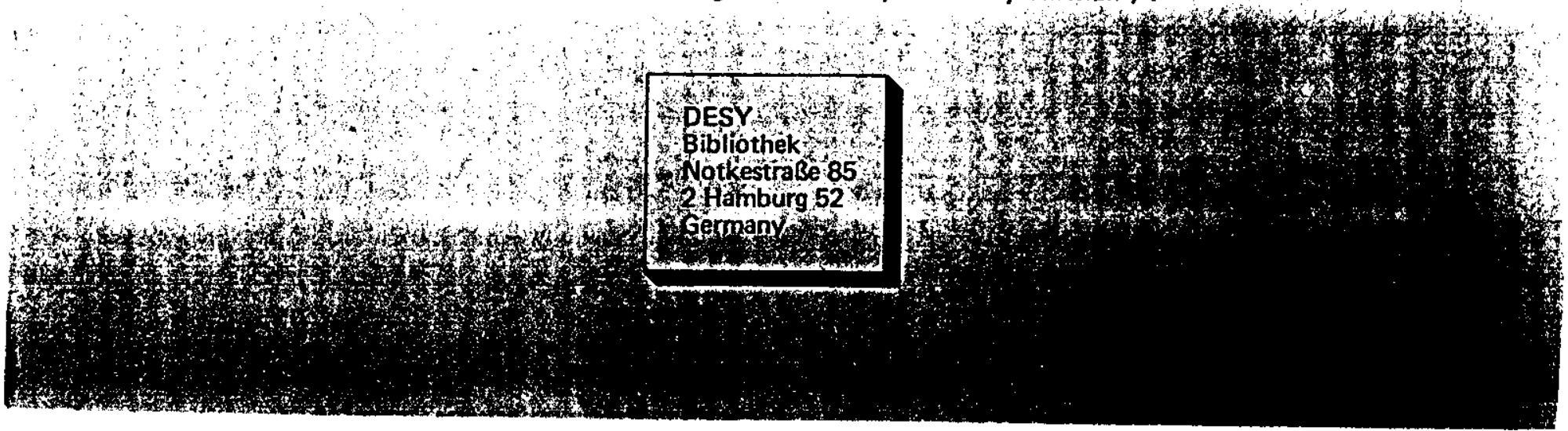


INCLUSIVE SPECTRA OF ELECTROPRODUCED $\mathrm{K}^{+}$-MESONS

H. Ackermann, T. Azemoon, W. Gabriel, D. Lüke ${ }^{+}$, H.D. Mertiens, H.D. Reich, G. Specht ${ }^{++}$

Deutsches Elektronen-Synchrotron DESY, Hamburg

\section{E. Ganssauge}

Universität Marburg

F. Janata, D. Schmidt

Gesamthochschule Wuppertal

\section{Abstract}

We report measurements of kaon electroproduction from a hydrogen target carried out at DESY. The invariant cross section for the reaction e $\mathrm{p} \rightarrow \mathrm{e} \mathrm{K}^{+}$ + anything is given as a function of the variables $q^{2}, w, x, p_{\perp}^{2}$ and $\phi$ in the following region:

$$
\begin{aligned}
-0.5 & <\mathrm{q}^{2}<-0.1 \mathrm{GeV}^{2} \\
2.0 & <\mathrm{W}<2.8 \mathrm{GeV} \\
0.3 & <\mathrm{x}<1.0 \\
\mathrm{P}^{2} & <0.25 \mathrm{GeV}^{2} \\
0^{\circ} & <\phi<360^{\circ}
\end{aligned}
$$

+ Now at SLAC, Stanford, Calif. 94305, USA

++ Now at Fachhochschule Hamburg

\section{Introduction}

One way to gain more insight into the hadronic structure of elementary particles is to study the final state hadrons in electron-scattering experiments at high energies. Here we present inclusive $\mathrm{K}^{+}-$distributions of the reaction

$$
\mathrm{e}^{-}+\mathrm{p} \rightarrow \mathrm{e}^{-}+\mathrm{K}^{+}+\text {anything }
$$

obtained by detecting the $\mathrm{K}^{+}$-meson in coincidence with the scattered electron in an electron-proton scattering experiment at $\mathrm{DESY}^{+}$.

In first order quantum-electrodynamics electroproduction can be regarded as photoproduction with virtual photons. The incident electron emits a virtual photon which interacts with the target proton. The unknown part is the reaction

$$
\gamma_{\mathrm{v}}+\mathrm{p} \rightarrow \mathrm{K}^{+}+\text {anything }
$$

where $\gamma_{\mathrm{v}}$ indicates the virtual photon.

To study the dynamical behaviour of this process and for comparison with photoproduction and pure hadronic reactions we show the dependence of the cross section on the kinematical variables $q^{2}, W, x, p_{1}^{2}$ and $\phi$ which are defined in the following way:

$q^{2}=\left(e-e^{\prime}\right)^{2} \quad$ is the four-momentum squared of the virtual photon. Here $e$ and $e^{\prime}$ are the four-momenta of the incoming and outgoing electrons respectively.

$W=\sqrt{(q+P)^{2}}$ is the centre of mass energy of the virtual photon and the target proton, where $q$ and $P$ indicate the four-momenta of the virtual photon and the target proton.

+ Some preliminary results on part of the reported data were presented at Bonn Conference $[1]$ where too high a normalization was used. 


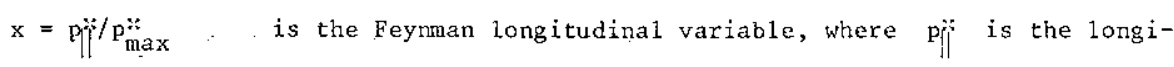
tudina1 momentun of the outgoing kaon with respect to the direction of the photon and $\mathrm{p}_{\max }^{: *}$ is the maximam momentum of the outgoing kaon at the observed value of $W$, which is obtained in the reaction $\gamma_{\mathrm{V}}+\mathrm{p} \rightarrow \mathrm{K}^{+}+\Lambda(1116)$. Both momenta refer to the c.m.s. of the photon and the target proton.

is the square of the transverse momentum of the outgoing kaon with respect to the direction of the photon.

is the azimuthal angle of the outgoing kaon, which is the anghe between the polarization plane, subtended by $\vec{e}$ and $\vec{e}$ ', and the production plane, subtended by $\vec{q}$ and the monentum p of the kaon.

As a consequence of the one-photon exchange mechanism the cross section of reaction ( 1 ) factorizes in $2 \pi \Gamma^{\prime}$, describing the flux of virtual photons, and the cross section $d \sigma / d^{3} p$ for reaction (2) [2],

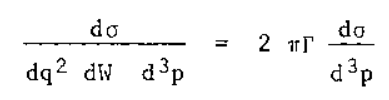

where $\Gamma$ is given by

$$
\Gamma=\frac{\alpha}{2(2 \pi)^{2}} \frac{W}{E_{0}^{2} M^{2}\left|q^{2}\right|} \frac{W^{2}-M^{2}}{1-\varepsilon} .
$$

Here $\mathrm{E}_{\mathrm{o}}$ is the energy of the primary electron in the 1aboratory frame, $M$ the mass of proton and $\varepsilon$ the degree of the transverse polarization of the photon

$$
\varepsilon=\frac{4 E_{O} E^{\prime}+q^{2}}{2 E_{O}^{2}+2 E^{\prime 2}-q^{2}}
$$

where $E^{\prime}$ is the energy of the scattered electron.

In this paper the differential cross section for virtual photoproduction is given in the Lorentz invariant form normalized with the total cross section

$$
\frac{F}{\sigma_{\text {tot }}} \cdot \frac{d \sigma}{d^{3} p}
$$

(E is the energy of the detected kaon. The values of $\sigma_{\text {tot }}$ were taken from ref. [3]).

In the framework of one-photon exchange the cross section can be parametrized as [4]

$$
\frac{\mathrm{E}}{{ }_{\text {tot }}^{\sigma} \cdot \frac{\mathrm{d} \sigma}{\mathrm{d}^{3} \mathrm{p}}} \div \mathrm{E}=\mathrm{f}_{\mathrm{u}}+\varepsilon \mathrm{f}_{\mathrm{L}}+\varepsilon \mathrm{f}_{\mathrm{p}} \cos 2 \phi+\sqrt{2 \varepsilon(\varepsilon+1)} \mathrm{f}_{1} \cos \phi
$$

The subscripted functions depend on $\mathrm{q}^{2}, \mathrm{~W}, \mathrm{x}$ and $\mathrm{p}_{1}^{2}$ only and they describe the contributions from the unpolarized transverse virtual photons, the longitudinal photons, the transverse linear polarization of the photons and the intercerence between the transverse and longitudinal components.

\section{Datat Analysis and Corrections}

The experimental details of the spectrometers which detected the scattered electron and the outgoing kaon can be found in ref. [5]. The separation of kaons, pions and protons by means of time of flight measurement system and a Cerenkov counter has been described in ref, [6]. To cover the $W$-interval from 2.0 to $2.8 \mathrm{GeV}$ and $\mathrm{q}^{2}$ fron -0.5 to $-0.1 \mathrm{GeV}^{2}$ data were taken at two different 
energies of the incident electron, $\mathrm{E}_{\mathrm{o}}=4.0 \mathrm{GeV}$ and $4.9 \mathrm{GeV}$. Corrections were made for the efficiency loss in the trigger counters, the electron cerenkov counter and shower counters, for the strong interaction of the kaons, the target walls and the loss in the automatic data analysis procedure.

The decay of the kaons was incorporated in the acceptance calculations. The kaons were defined by appropriate cuts in the time of flight spectra, which leads to momentum dependent corrections. Also we had to correct for the background of pions and protons within the kaon cuts at each point where the cross section was calculated. The background corrections vary between $1 \%$ and $20 \%$.

The uncertainties in all these corrections including that in the intensity of the primary beam add up to an overall systematic error of less than $8 \%$ which is not included in the errors given in this report. Neither have the radiative corrections been taken into account, which are of the order of a few per cent.

\section{Results}

The accepted range of the azimuthal angle $\phi$ depends on the variables $q^{2}$

and $w$. Therefore the data were analysed in four distinct $\left(q^{2}, W\right)$-regions with the following limits in $\phi$ :

$\begin{array}{rlrl}\text { Region A } & -60^{\circ} & <\phi<60^{\circ} \\ \text { Region B } & -90^{\circ} & <\phi<90^{\circ} \\ \text { Region C } & 0^{\circ}<\phi<360^{\circ} \\ \text { Region E } & 90^{\circ}<\phi<270^{\circ}\end{array}$

We calculated the invariant cross section $\mathrm{E} / \sigma_{\text {tot }} \cdot \mathrm{d} \sigma / \mathrm{d}^{3} \mathrm{p}$ averaged over the accepted $\phi$-range. The values are given in tables $2-5$.

In region $C$ the $\phi$-dependence of the cross section was studied according to (4) at two different $\left(q^{2}, W\right)$-points. The result is given in table 1 . Compared with $f_{u}+\varepsilon f_{L}$ the polarization term $f_{p}$ and the interference term $f_{I}$ are small ( $10 \%$ to $20 \%$ of $\left.f_{u}+E^{\prime} f_{L}\right)$. Thus the important term is the $\phi$-independent part of the cross section. Assuming a similar behaviour in the other $\left(\mathrm{q}^{2}, \mathrm{~W}\right)$-regions the polarization term and interference term multiplied by the appropriate factors, that is the $\phi$-average of $\varepsilon \cos 2 \phi$ and $\sqrt{2 \varepsilon(\varepsilon+1)}$ respectively, can be ignored, enabling one to combine the data from all regions.

Fig. I shows the $x$-dependence at $W=2.65 \mathrm{GeV}$ and $q^{2}=-0.23 \mathrm{GeV}^{2}$ for different transverse momenta. By comparison with the data of pion production [7], which range from 0.5 to $0.3 \mathrm{GeV}^{-2}$ in the $\mathrm{x}$-interval from 0.5 to 0.8 , we see that the kaon-cross section is an order of magnitude smaller than the pion cross section. The same ratio between the $\mathrm{K}^{+}-$and $\pi^{+}$-production was observed in photoproduction [8] and in pp-scattering [9]. The kaon-electroproduction experiment of Alder et al. [10] yields, in forward direction at $q^{2}=-1.15 \mathrm{GeV}^{2}$ and $w=2.65 \mathrm{GeV}$, slightly higher values, about $0.07 \mathrm{GeV}^{-2}$, than ours at $\mathrm{q}^{2}=-0.23 \mathrm{GeV}^{2}$.

Keeping $x$ fixed the cross section falls with $p_{1}^{2}$. In fig. 2 the dependence of the cross section on $\mathrm{P}^{2}$ is plotted at $\mathrm{W}=2.65 \mathrm{GeV}$ and $\mathrm{q}^{2}=-0.23$ $\mathrm{GeV}^{2}$. The line is an exponential form $\mathrm{e}^{-\mathrm{ap}} \mathrm{p}^{2}$ with a slope of $\mathrm{a}=5 \mathrm{GeV}^{-2}$ which fits the data well. Only the $0.6<x<0.7$-region shows a different behaviour. This can be explained by the dominance of the $K^{+}+\Lambda(1520)$-channel which in this $x$-region can only contribute to $p_{1}^{2}>0.1 \mathrm{GeV}^{2}$. An exponential distribution with a slope of $5 \mathrm{GeV}^{-2}$ corresponds to a mean $\mathrm{P}_{\perp}$ of $0.4 \mathrm{GeV}$. Similar mean transverse momenta have been found in photoproduction of kaons [8] and in pure hadronic reactions 1 ike $\pi^{+}+p \rightarrow K^{+}+$anything $[11]$ and $\mathrm{p}+\mathrm{p} \rightarrow \mathrm{K}^{+}+$anything $[9]$.

At small $x(0.4<x<0.6)$, the cross section is nearly constant from $W=2.2$ to $2.8 \mathrm{GeV}$ (fig. 3). Bebek et al. [12] a1so have seen no variation 
in the cross section between $\mathrm{W}=2.2$ and $3.1 \mathrm{GeV}$ at $\mathrm{q}^{2}=-1.2 \mathrm{GeV}^{2}$ and $0.2<x<0.6$. This implies that Feyman scaling roughly works for smaller $x$ even at such low energies. But at high $x(0.8<x<1.0)$ the cross section is rapidly decreasing with increasing $x$, see fig. 4. The larger values of pI for the high w-points can explain only a small amount of this decrease. Since only the production of $\Lambda(1116), \Sigma(1193), \Sigma(1385)$ and $\Lambda(1405)$ contributes to this $x$-region, the slope can easily be explained by decreasing production of these exclusive channels.

In figs. 5 and 6 the $q^{2}$-dependence is shown at $W=2.43$ and $2.65 \mathrm{GeV}$ for different $x$-intervals. The lines in the figures correspond to the mean of all points of the same $x$-interval. The cross section is constant or weakly rising. Since the $\pi^{+}$-production falls by a factor of two from $\mathrm{q}^{2}=-0.1$ to $-0.6 \mathrm{GeV}^{2}$ at $W=2.65 \mathrm{GeV}[7]$, this $\mathrm{q}^{2}$-behaviour supports the quark-parton model [13] which predicts a rising $\mathrm{K}^{+}$- over $\pi^{+}$- ratio with increasing $\left|\mathrm{q}^{2}\right|$.

\section{Sumnary}

The exponential decrease of kaon production with $\mathrm{p}_{1}^{2}$ shows a similar slope as found in photoproduction and in pure hadronic reactions. At high $x$ the cross section strongly decreases with rising energy $W$ due to non-diffractive twobody channels. But at smaller $\mathrm{x}$ we see a nearly $\mathrm{w}$-independent behaviour. The invariant cross section normalized with $\sigma_{\text {tot }}$ shows a flat $\mathrm{q}^{2}$-behaviour.

\section{Acknowledgements}

Some of us (E. Ganßauge, F. Janata and D. Schmidt) would like to thank Profes- , sors H. Schopper and G. Weber for the kind hospitality at DESY.

We are indebted to Professor K. Heinloth and Drs. I. Dammann and C. Driver for their valuabile assistance during the data collection.

We also want to thank Mr. G. Augustinski, Mr. P. Burmeister, Mr. G. Hase, Ar. K. Maschidlauskas and Mrs. R. Siemer for their excellent assistance. The valuable cooperation of the Synchrotron crew, the Hallendienst; the Kältetechnik and the Rechenzentrum is gratefully acknowledged. 
References

[1] T. Azemoon, I. Dammann, C. Driver, K. Heinloth, F. Janata, D. Lüke, D. Schmidt, G. Specht, H. Ackermann, E. Ganssauge,

Contribution to the International Symposium on Electron and Photon Interactions at High Energies, Bonn, 1973.

[2] L.N. Hand,

Phys. Rev. 129 (1963) 1834.

[3] F.W. Brasse, E. Chazelas, W. Fehrenbach, K.H. Frank, E. Ganssauge, J. Gayler, V. Korbe1, J. May, M. Merkwitz, V. Rittenberg,

H.R. Rubinstein,

Nucl. Phys. B39 (1972) 421 .

[4] S.M. Berman,

Phys. Rev. 135 (1964) 1249.

[5] C. Driver, K. Heinloth, K. Höhne, G. Hofmann, P. Karow, D. Schmidt, G. Specht, J. Rathje,

Nuc1. Phys. B30 (1971) 245.

[6] T. Azemoon, I. Dammann, C. Driver, D. Lüke, G. Specht, X. Heinloth, H. Ackermann, E. Ganssauge, F. Janata, D. Schmidt, Nuc1. Phys. B95 (1975) 77.

[7] I. Dammann, C. Driver, K. Heinloth, G. Hofmann, F. Janata, P. Karow, D. Lüke, D. Schmidt, G. Specht, Nuc1. Phys. B54 (1973) 381.

[8] H. Burfeindt, G. Buschhorn, H. Genzel, P. Heide, U. Kötz, K.-H. Mess, P. Schmüser, B. Sonne, G. Vogel, B.K. Wiik, Nucl. Phys. B74 (1974) 189.
[9] A. Bertin, P. Capiluppi, M. D'Agostini-Bruno, G. Giacome11i, A.M. Rossi, G. Vannini, A. Bussière, R.T. Poe, Phys. Letters 42B (1972) 493.

[10] J.C. Alder, F.W. Brasse, E. Chazelas, W. Fehrenbach, W. Flauger, K.H. Frank, E. Ganssauge, J. Gayler, V. Korbel, W. Krechlok, J. May, M. Merkwitz, P.D. Zimmermann, Nucl. Phys. B46 (1972) 415.

111] V.v. Glagolev, G.D. Restova, Dubna Preprint ITNR-D-1-9093 (1975).

[12] C.J. Bebek, C.N. Brown, P. Bucksbaum, M. Herzlinger, S.D. Holmes, C.A. Lichtenstein, F.M. Pipkin, S.W. Raither, L.K. Sisterson, Phys. Rev. D15 (1977) 594.

[13] M. Perl,

Proceedings of Sumner Institute of Particle Physics, Stanford, 1973, 35 . 


\section{Figure Captions}

Fig. I The $\mathrm{x}$-distribution for $\mathrm{W}=2.65 \mathrm{GeV}$ at different transverse momenta.

Fig. 2 The $\mathrm{p}^{2}$-distribution for $\mathrm{W}=2.65 \mathrm{GeV}$ at different $\mathrm{x}$-values. The solid lines correpond to an exponential with a slope of $5 \mathrm{GeV}^{-2}$

Fig. 3 The W-dependence for small $x$. The full symbols belong to $\mathrm{E}_{\mathrm{o}}=4.0 \mathrm{GeV}$, the open symbo1s to $\mathrm{E}_{\mathrm{O}}=4.9 \mathrm{GeV}$.

$0: \mathrm{q}^{2}=-0.5 \mathrm{GeV}^{2}, \quad 0.0<\mathrm{p}^{2}<0.02 \mathrm{GeV}^{2}, \quad 0^{\circ}<\phi<360^{\circ}$

$\triangle: q^{2}=-0.4 \mathrm{GeV}^{2}, \quad 0.0<\mathrm{p}^{2}<0.02 \mathrm{GeV}^{2},-90^{\circ}<\phi<90^{\circ}$

$\square: \mathrm{q}^{2}=-0.3 \mathrm{GeV}^{2}, \quad 0.0<\mathrm{p}_{1}^{2}<0.04 \mathrm{GeV}^{2},-60^{\circ}<\phi<60^{\circ}$

$\boldsymbol{\nabla}: \quad \mathrm{q}^{2}=-0.18 \mathrm{GeV}^{2}, \quad 0.0<\mathrm{p}^{2}<0.025 \mathrm{GeV}^{2},-60^{\circ}<\phi<60^{\circ}$

$\nabla: \quad q^{2}=-0.18 \mathrm{GeV}^{2}, 0.025<\mathrm{p}^{2}<0.1 \mathrm{GeV}^{2},-60^{\circ}<\phi<60^{\circ}$

Fig. 4 The W-dependence for high $x$. The full symbols belong to $\mathrm{E}_{\mathrm{o}}=4.0 \mathrm{GeV}$, the open symbols to $E_{\mathrm{o}}=4.9 \mathrm{GeV}$.
O: $\quad q^{2}=-0.5 \mathrm{GeV}^{2}, \quad 0.0<\mathrm{p}^{2}<0.05 \mathrm{GeV}^{2}, \quad 0^{\circ}<\phi<360^{\circ}$
$\Delta: \mathrm{q}^{2}=-0.4 \mathrm{GeV}^{2}, \quad 0.0<\mathrm{p}^{2}<0.04 \mathrm{GeV}^{2}, \quad 90^{\circ}<\phi<270^{\circ}$
$\triangle: \quad q^{2}=-0.4 \mathrm{GeV}^{2}, \quad 0.01<p_{L}^{2}<0.07 \mathrm{GeV}^{2},-90^{\circ}<\phi<90^{\circ}$
D : $\mathrm{q}^{2}=-0.3 \mathrm{GeV}^{2}, \quad 0.0<\mathrm{p}^{2}<0.04 \mathrm{GeV}^{2}, \quad 0^{\circ}<\phi<360^{\circ}$
$\square: \mathrm{q}^{2}=-0.3 \mathrm{GeV}^{2}, 0.03<\mathrm{p}^{2}<0.11 \mathrm{GeV}^{2},-60^{\circ}<\phi<60^{\circ}$
$\nabla: \mathrm{q}^{2}=-0.18 \mathrm{GeV}^{2}, 0.0<\mathrm{p}^{2}<0.07 \mathrm{GeV}^{2},-60^{\circ}<\phi<60^{\circ}$
$\nabla: \quad \mathrm{q}^{2}=-0.18 \mathrm{GeV}^{2}, \quad 0.1<\mathrm{p}^{2}<0.25 \mathrm{GeV}^{2},-60^{\circ}<\phi<60^{\circ}$

Fig. 5 The $\mathrm{q}^{2}$-dependence for $\mathrm{W}=2.43 \mathrm{GeV}$. The 1 ines correspond to the mean value of the points with the sane $x$-value.

$$
\begin{array}{ll}
\boldsymbol{\square}: 0.4<\mathrm{x}<0.6, & \mathrm{p}_{1}^{2}<0.04 \mathrm{GeV}^{2} \\
\Delta: 0.6<\mathrm{x}<0.8, & \mathrm{p}_{1}^{2}<0.05 \mathrm{GeV}^{2} \\
\mathbf{0}: 0.8<\mathrm{x}<1.0, & \mathrm{p}_{1}^{2}<0.1 \quad \mathrm{GeV}^{2}
\end{array}
$$

Fig. 6 The $q^{2}$-dependence for $W=2.65 \mathrm{GeV}$. The lines correspond to the mean value of the points with the same x-value.

$$
\begin{array}{ll}
\nabla: 0.3<\mathrm{x}<0.4, & 0.005<\mathrm{p}_{1}^{2}<0.035 \mathrm{GeV}^{2} \\
\mathbf{\square}: 0.4<\mathrm{x}<0.6, & 0.015<\mathrm{p}_{1}^{2}<0.075 \mathrm{GeV}^{2} \\
\triangle: 0.6<\mathrm{x}<0.8, & 0.03<\mathrm{p}_{1}^{2}<0.1 \mathrm{GeV}^{2} \\
\mathbf{0}: 0.8<\mathrm{x}<1.0, & 0.05<\mathrm{p}_{1}^{2}<0.2 \mathrm{GeV}^{2}
\end{array}
$$


Table Captions

Table 1 The values of $f_{u}+\varepsilon f_{L}, f_{p}$ and $f_{I}$ at small $p_{1}^{2}$ $\left(\mathrm{p}^{2}<0.01 \mathrm{GeV}^{2}\right)$.

Table 2 The $q^{2}$-dependence.

Table 3 The $w$-dependence.

Tab1e 4 The $x$-dependence.

Tab1e 5 The $\mathrm{p}^{2}$-dependence.

Table 6 The $\phi$-dependence.

Abbrreviations and definitions used_in tables_2 to 6

н

mean of the kinematical interval.

total width of the interval.

mean of the interval weighted by the acceptance.

$<>$ $\phi$-region.

$\alpha \equiv \varepsilon \frac{1}{\Delta \phi} \int_{\Delta \phi} \cos (2 \phi) \mathrm{d} \phi$

$\beta \equiv \sqrt{2 \varepsilon(\varepsilon+1)} \frac{1}{\Delta \phi} \int_{\Delta \phi} \cos \phi d \phi$

$\sigma_{t} \equiv \sigma_{\text {tot }}$

total cross section for virtual photons.

$\delta \mathrm{f}$

$m_{\mathrm{x}}$

$t \equiv(q-p)^{2}$

invariant mass of the unobserved particles. error on $\mathrm{f}$.

square of the four-momentum transfer from the

photon to the kaon. 


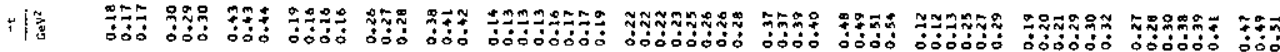

ه

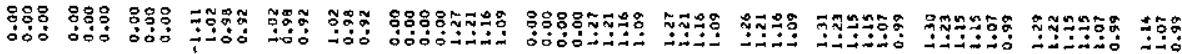

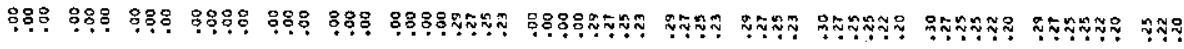

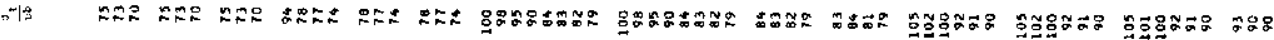

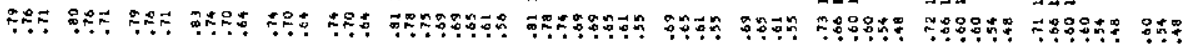

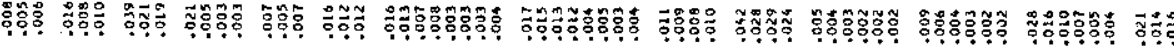

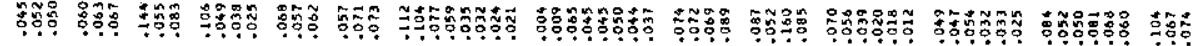

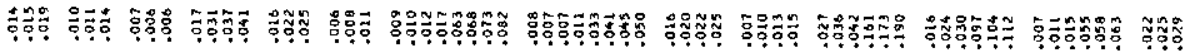

管 =

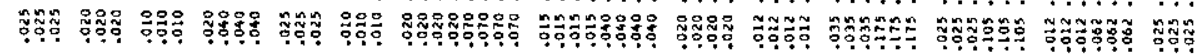

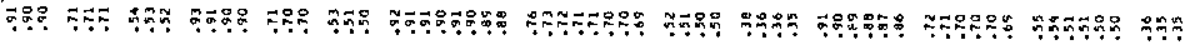
דר ד צ.

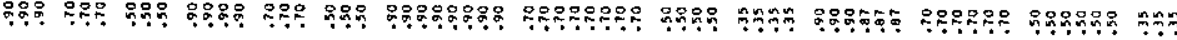

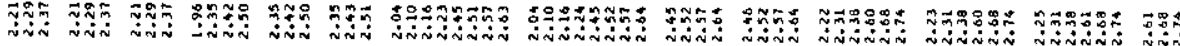

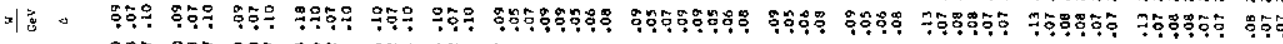

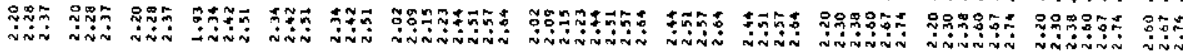

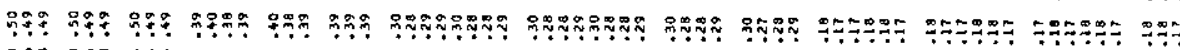

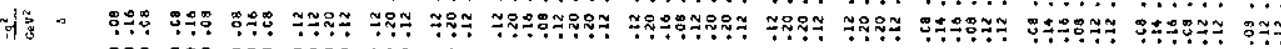

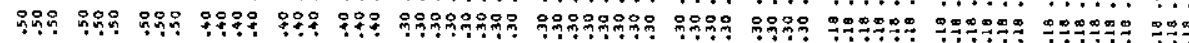

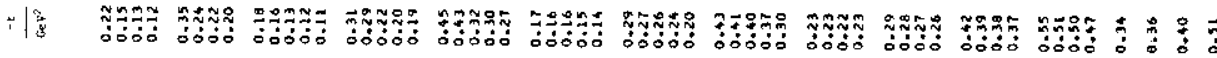

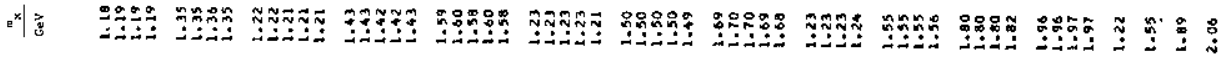

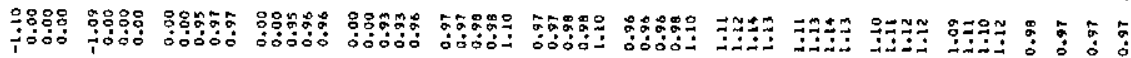

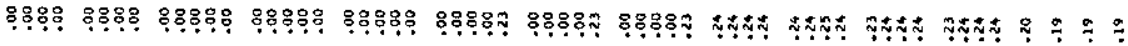

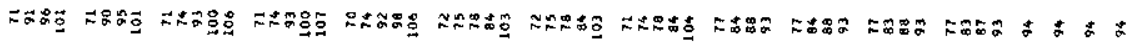

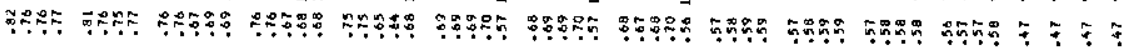

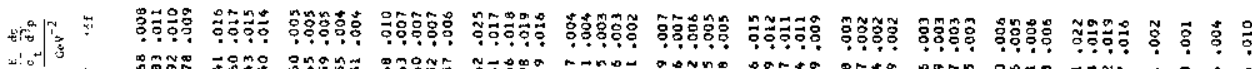

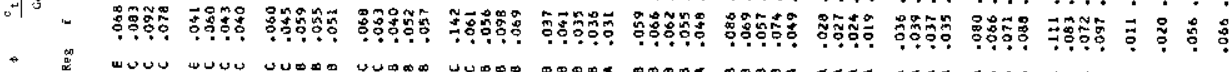

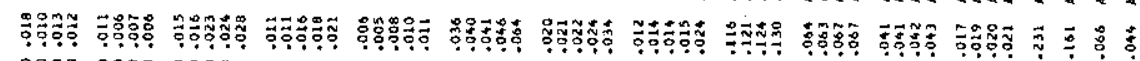

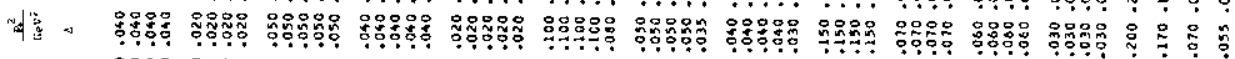

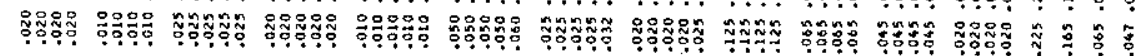

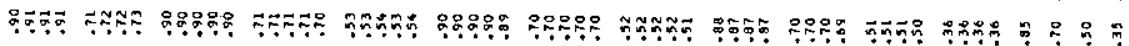
ت

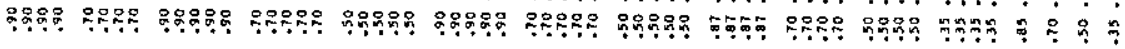

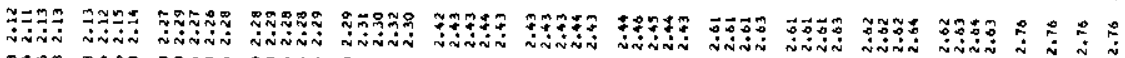

|

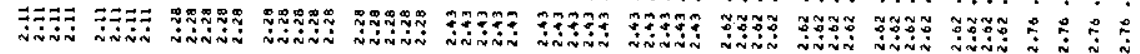

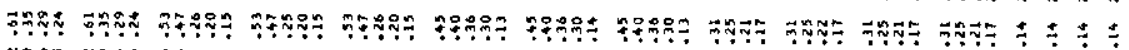

لَّ

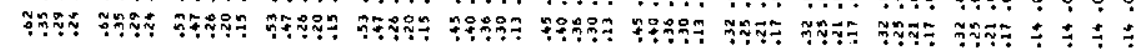




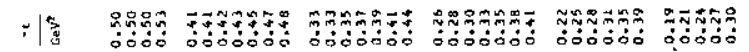

* 音

-

-

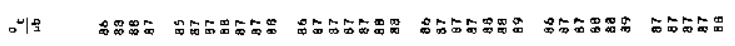

-

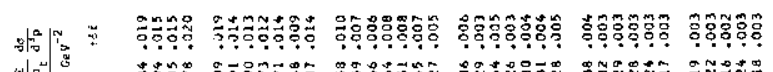

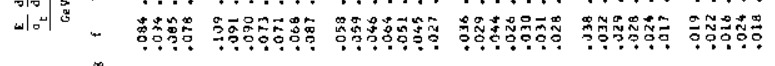

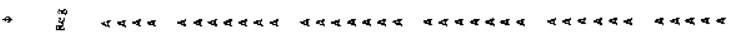

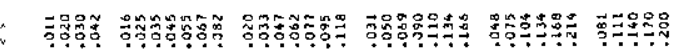

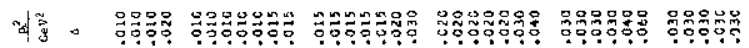

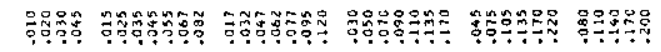

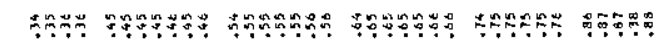

$\times$ a -

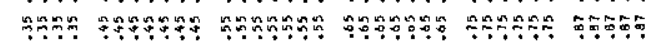

\&

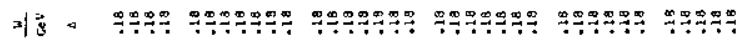

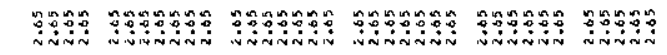

:

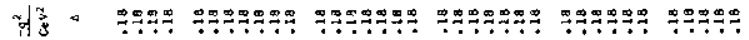

=

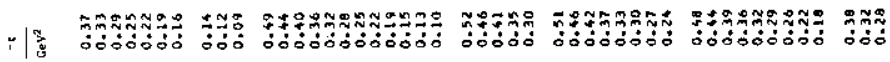

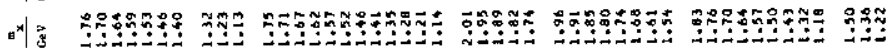

a

-

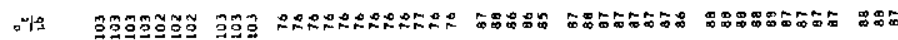

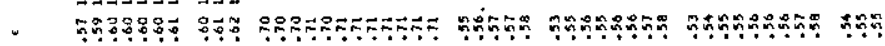

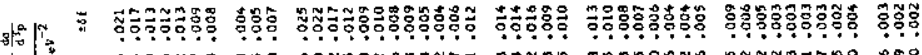
whots -

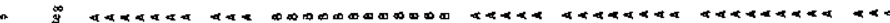

;

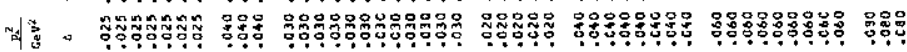

-

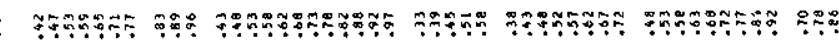

* -

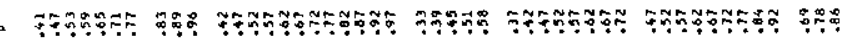

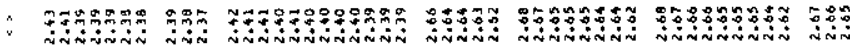

ג䧄

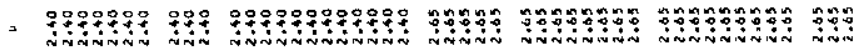

:

ل

= 


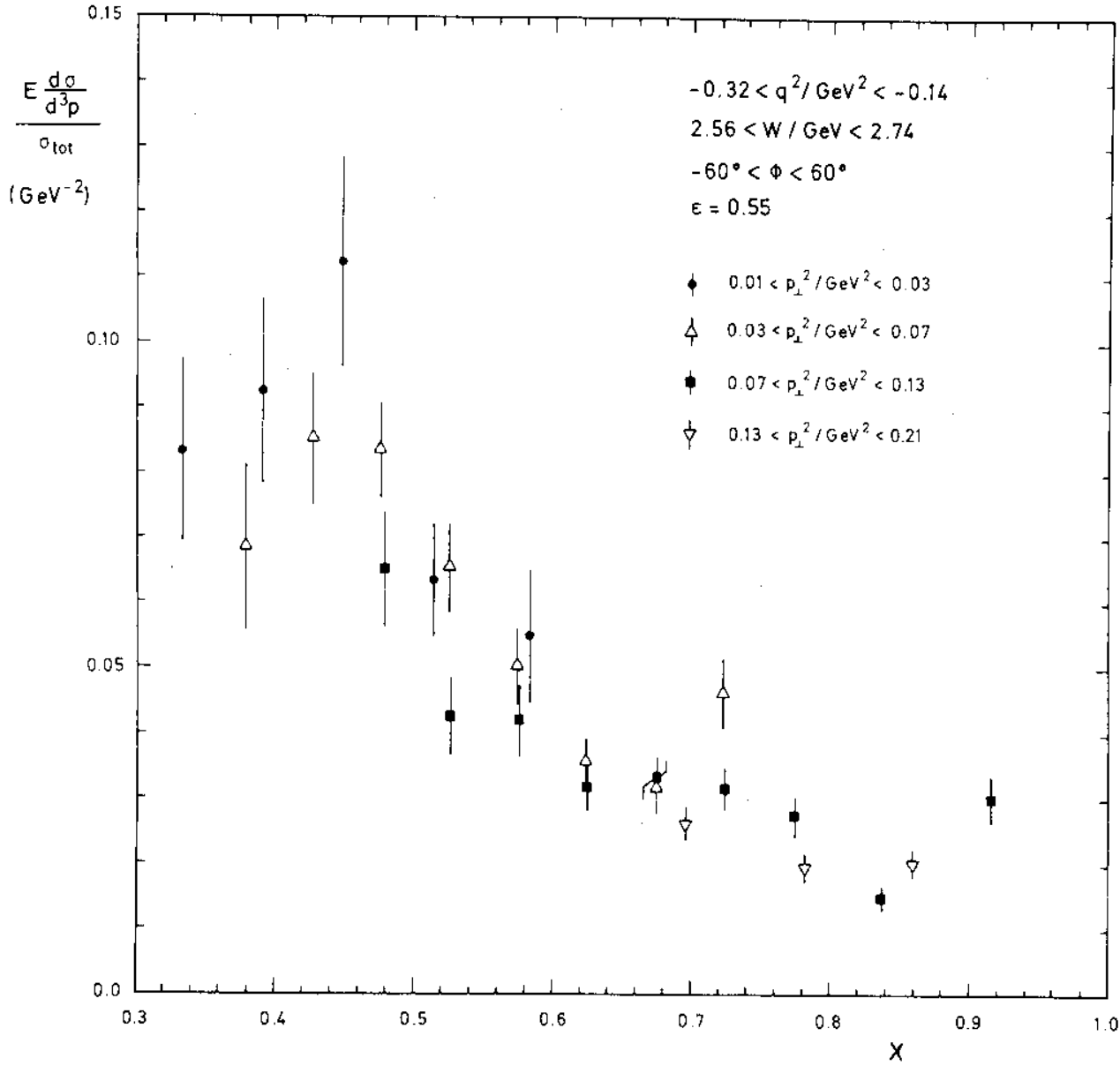

Fig. 1

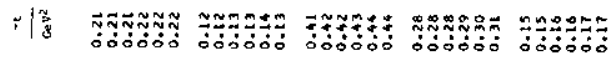

A 4 章

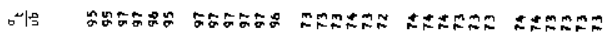

-

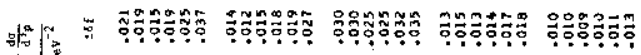

- -

:

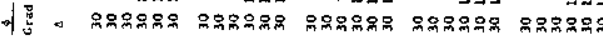

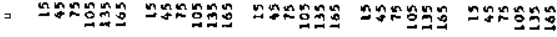

:

将等

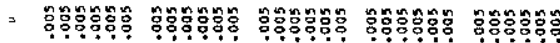

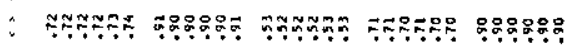

* 4 ม

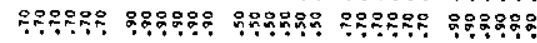

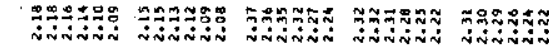

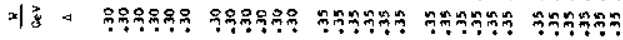

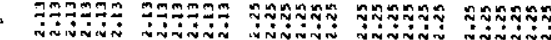

:

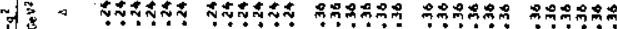

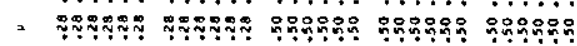



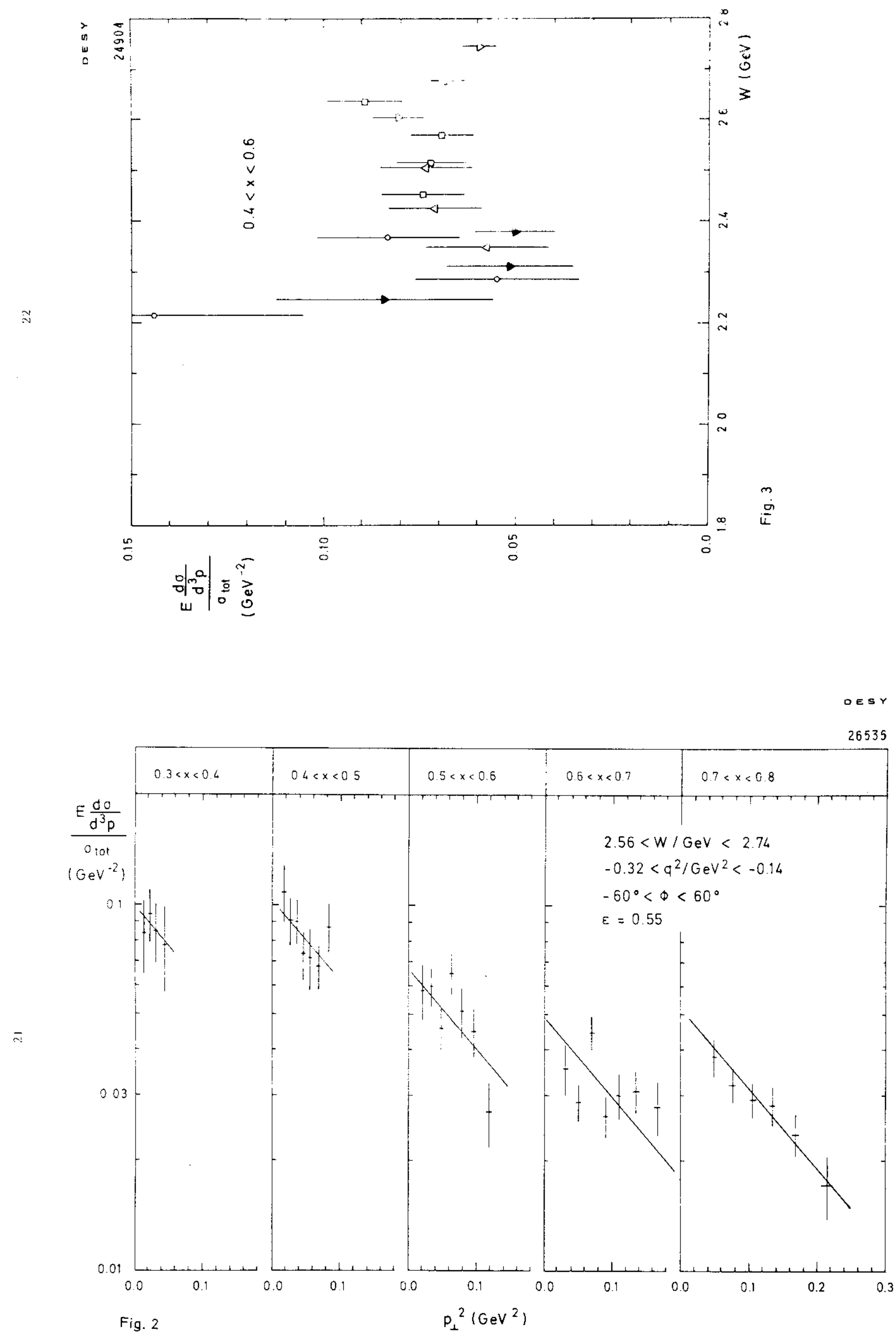


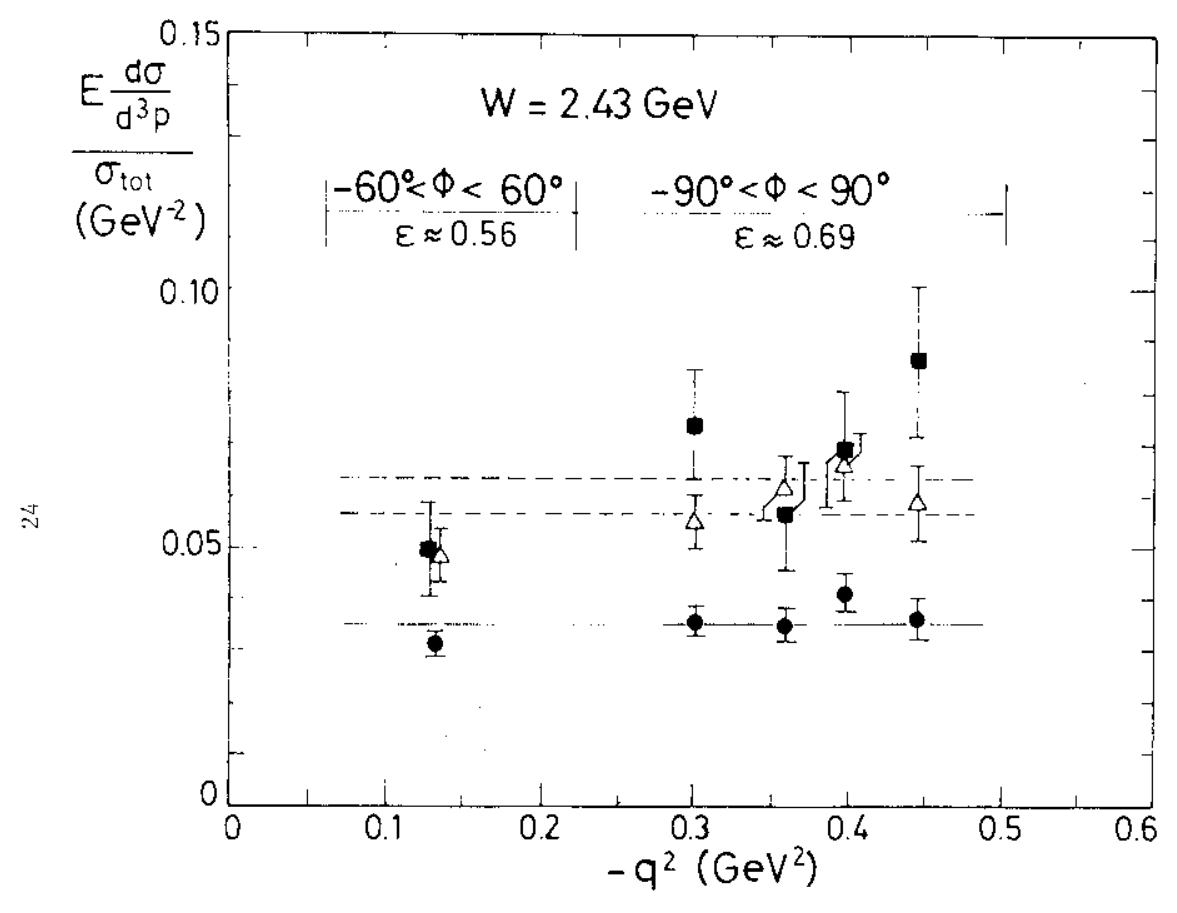

Fig. 5

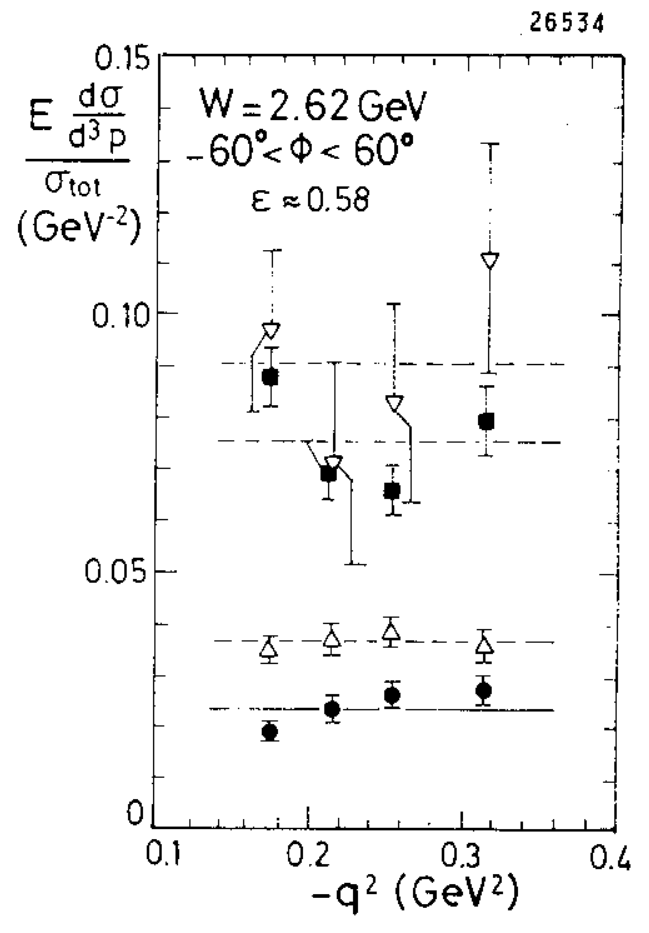

Fig. 6

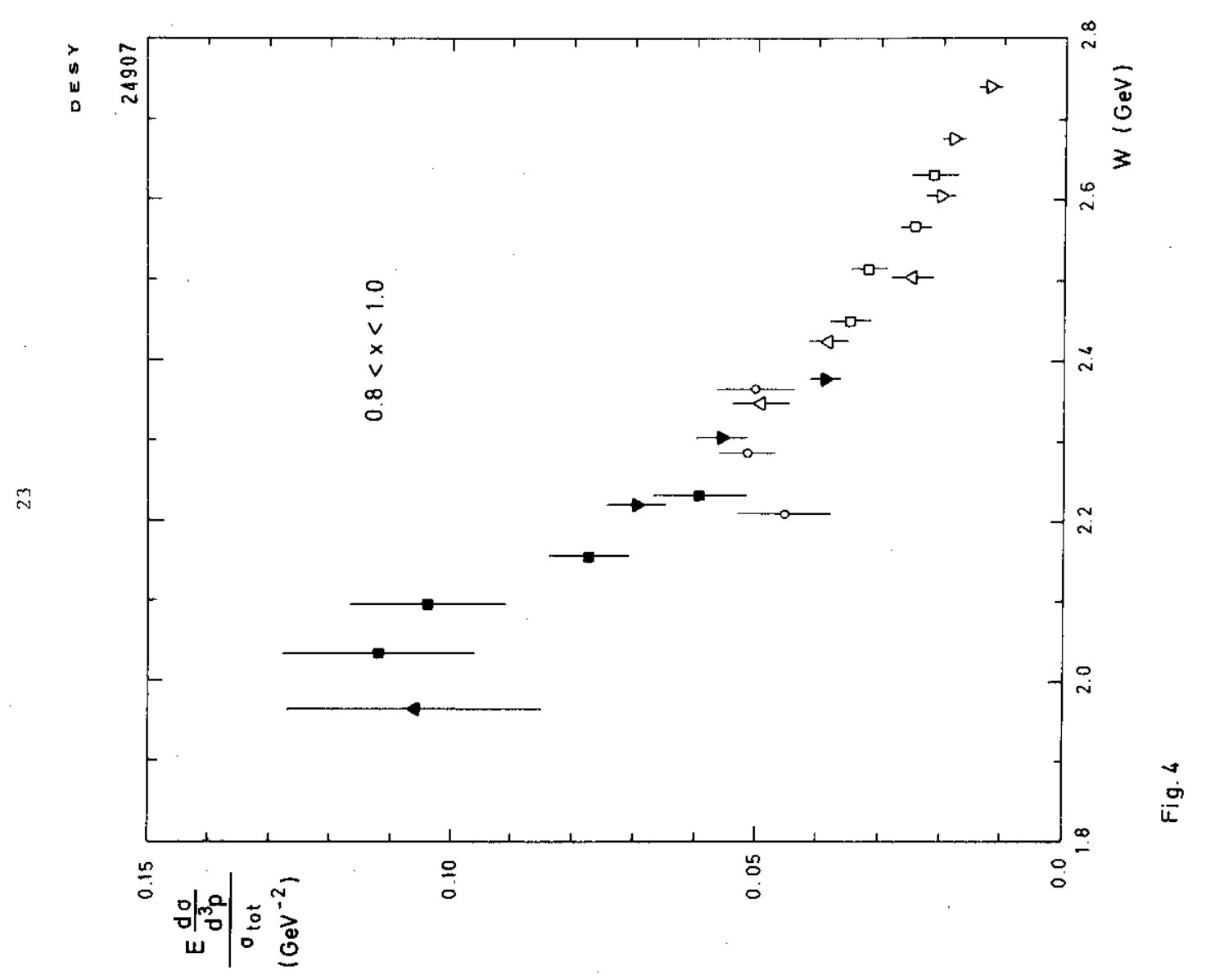

